Supporting Information

\title{
Unusual photoelectrochemical properties of electropolymerized films of a triphenylamine- containing organic small molecule
}

Hong Yin, Tong Yang, and Ke-Zhi Wang*

Beijing Key Laboratory of Energy Conversion and Storage Materials, College of Chemistry, Beijing Normal University, Beijing 100875, P.R. China

Jin Tong, and Shu-Yan Yu

Beijing Key Laboratory for Green Catalysis and Separation, Laboratory for

Self-Assembly Chemistry, Department of Chemistry and Chemical

Industry, College of Environmental and Energy Engineering, Beijing University of Technology, Beijing 100124, China 


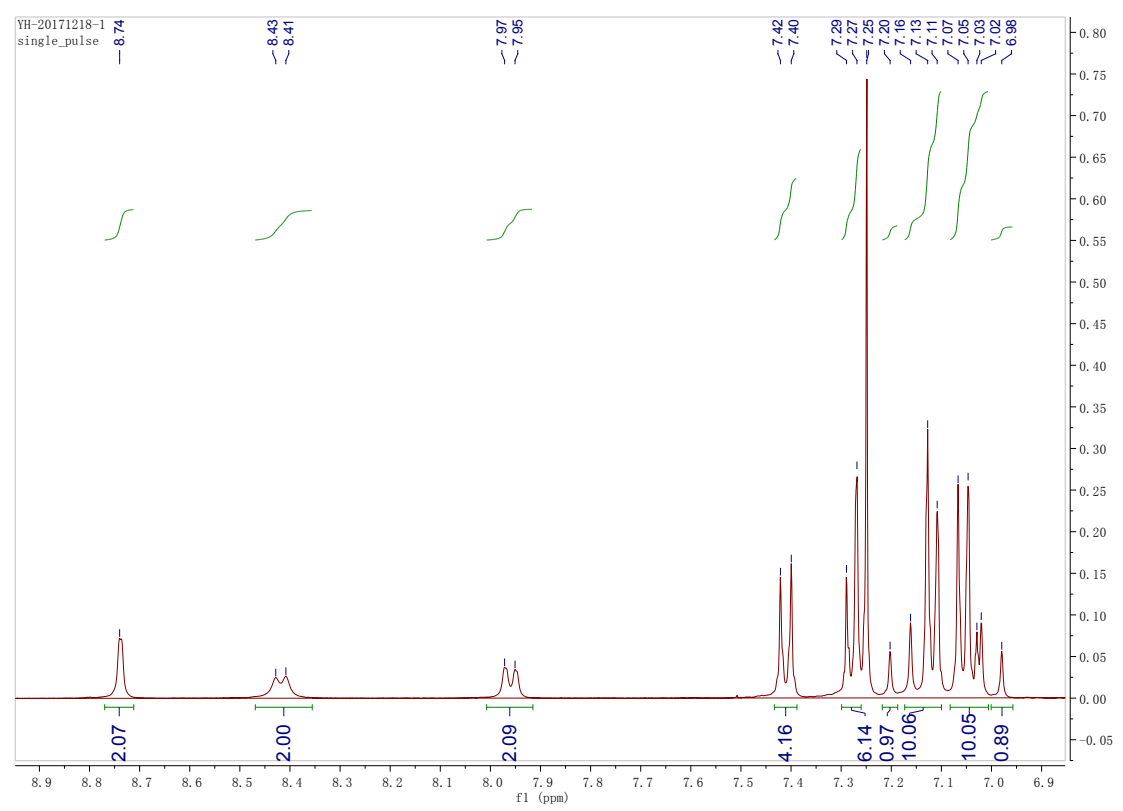

Figure S1. ${ }^{1} \mathrm{H}$ NMR spectrum of $L$. 

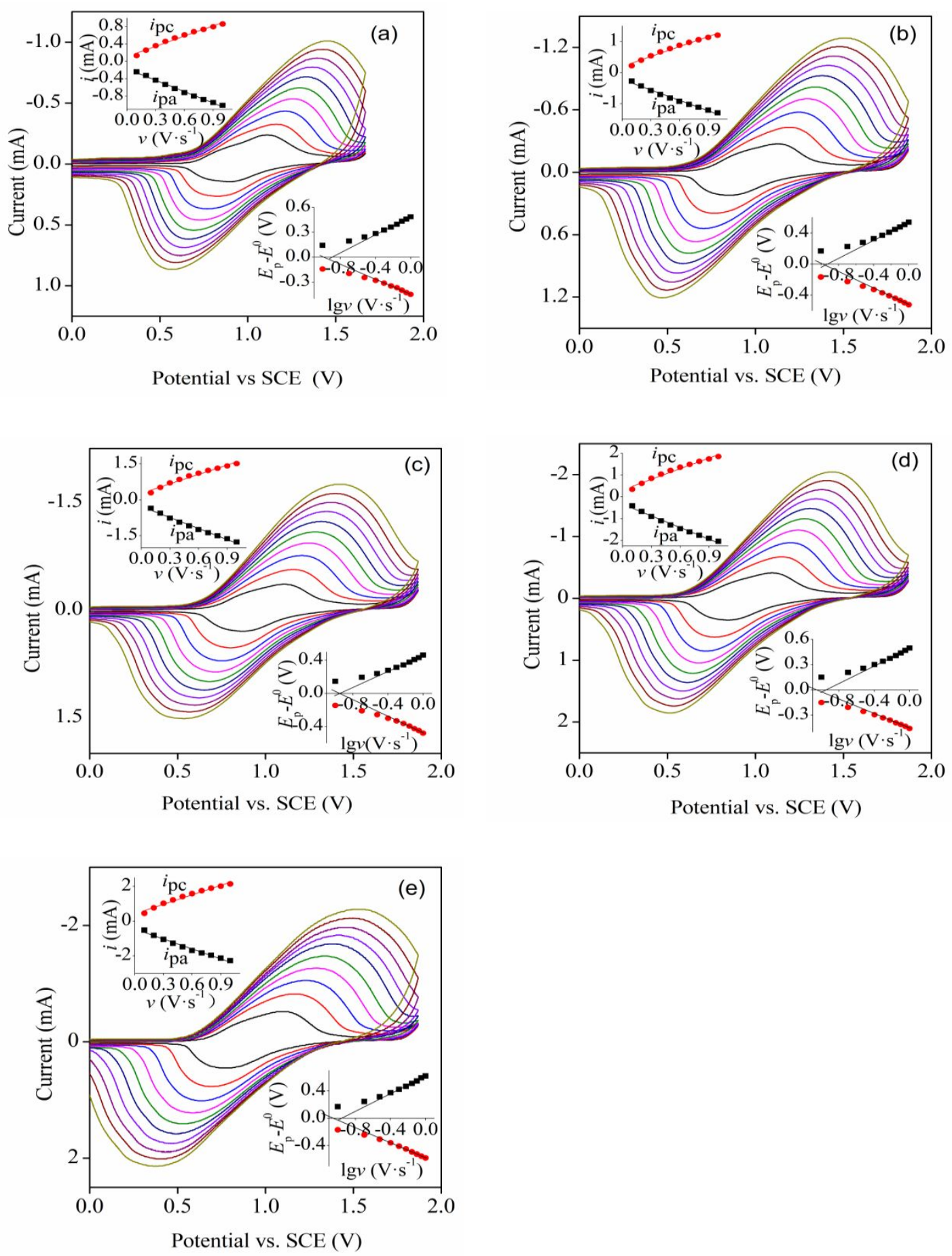

Figure S2. Cyclic voltammograms (a-e) of poly(L) ${ }_{n}(n=2$ (a), 3 (b), 4 (c), 5(d) and 6 (e)) films in $\mathrm{CH}_{2} \mathrm{Cl}_{2}$ solution that were recorded upon increasing potential scan rate $(v)$ from 0.1 to $1.0 \mathrm{~V} / \mathrm{s}$; Top left and bottom right insets of each figures show a polt of linear dependence of the peak currents vs. $v$, and 
a plot of the dependence of the cathodic and anodic overpotentials on $v$, respectively.

(a)

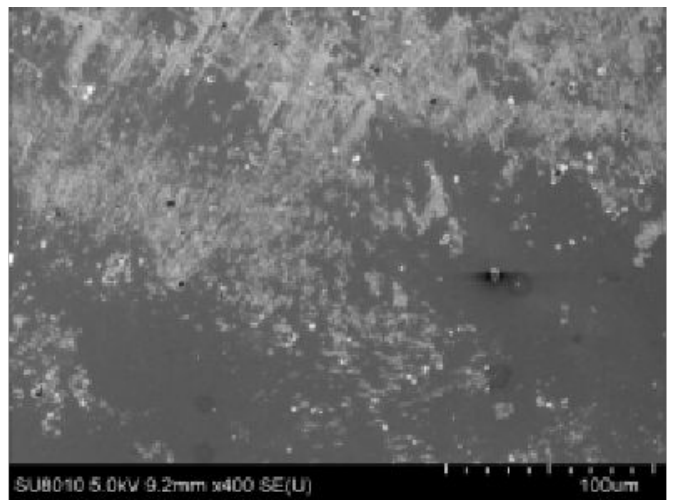

(b)

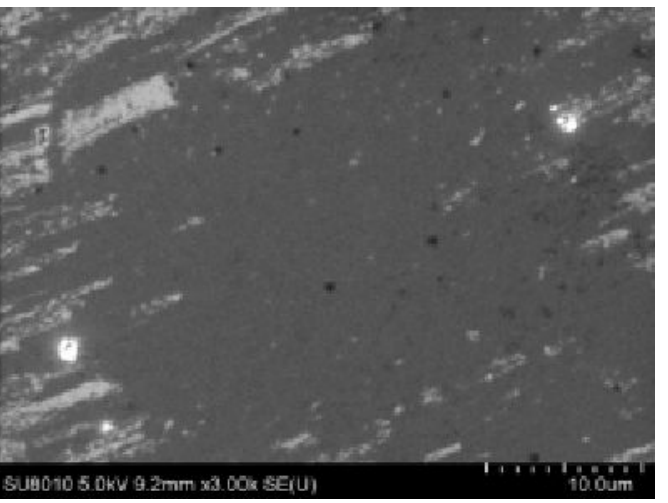


(c)

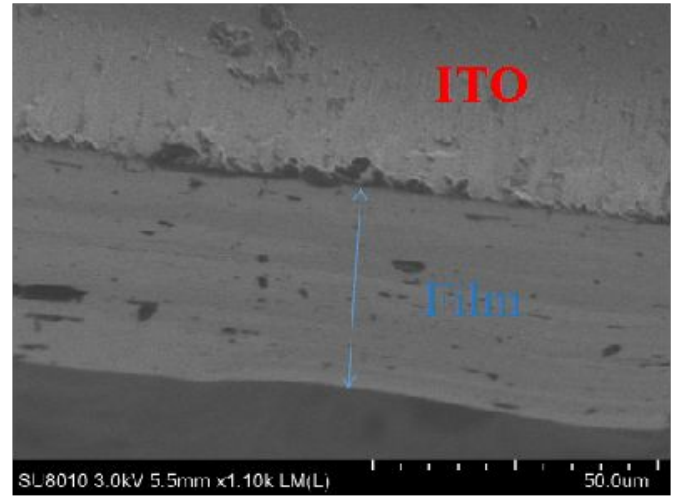

Figure S3. The surface morphology of poly $(L)_{20}(a, b)$ and cross section of $\operatorname{poly}(\mathrm{L})_{5}(\mathrm{C})$. 


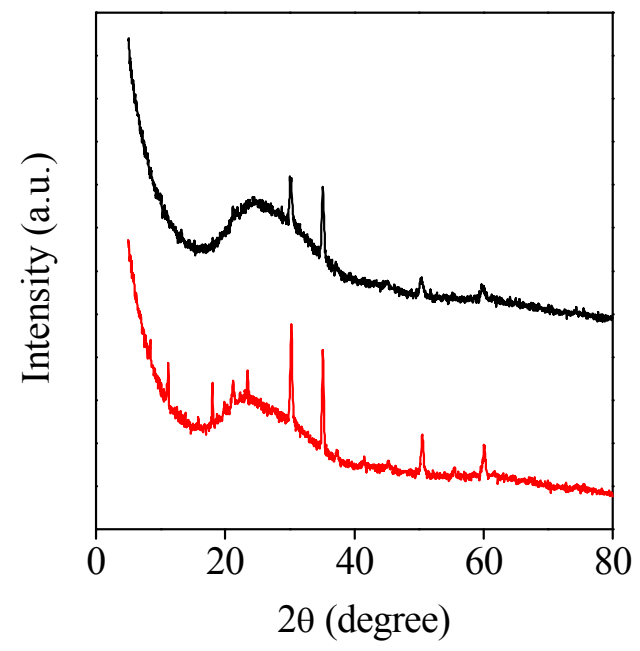

Figure S4. XRD patterns of bare ITO (top) and poly(L) $)_{20}$ film on ITO (bottom). 

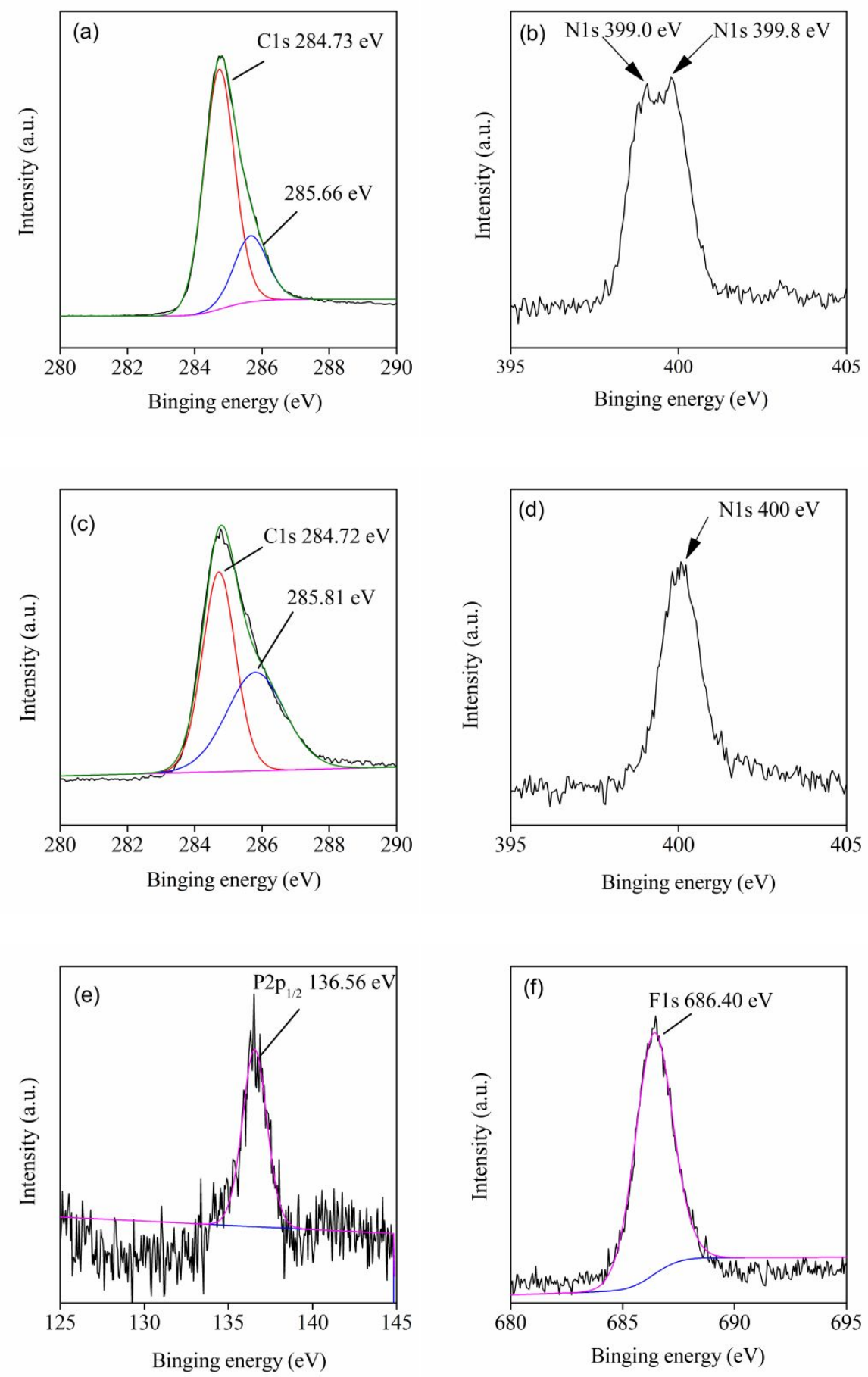
Figure S5. Highly resolved C1s (a) and N1s (b) XPS spectra of L powder; Highly resolved C1s (c), N1s (d), P2 $p_{1 / 2}$ (e) and F1s (f) XPS spectra of poly $(\mathbf{L})_{20}$ film.

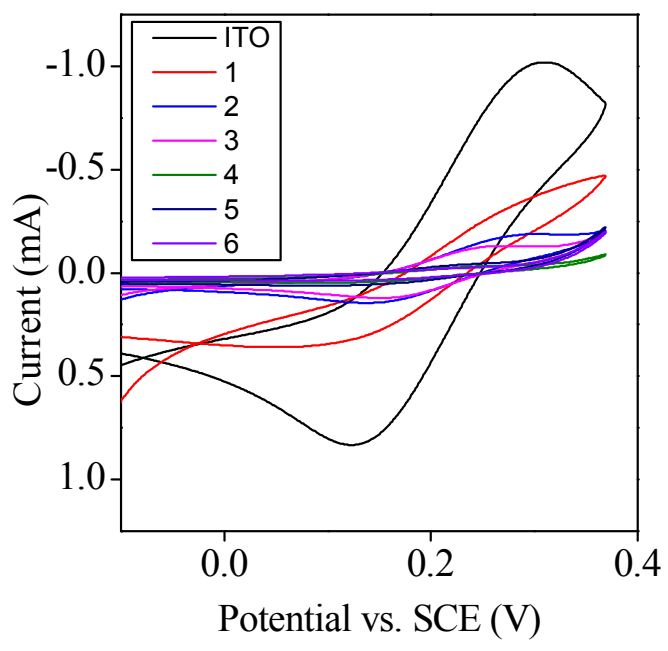

Figure S6. Cyclic voltammograms of blank ITO and electropolymerized film poly $(\mathrm{L})_{n}(n=1-6)$ in $0.1 \mathrm{M} \mathrm{HCl}$ solution. 